\title{
New insights into the tonoplast architecture of plant vacuoles and vacuolar dynamics during osmotic stress Daniel Reisen ${ }^{1,2}$, Francis Marty ${ }^{1}$ and Nathalie Leborgne-Castel*1
}

\author{
Address: ${ }^{1}$ UMR PME INRA/CNRS/Université de Bourgogne BP 47870, boulevard Gabriel, 21078 Dijon Cedex, France and ${ }^{2}$ Department of \\ Molecular Biology and Genetics, 321 Biotechnology Building, Cornell University, Ithaca, NY 14853, USA \\ Email: Daniel Reisen -dr237@cornell.edu; Francis Marty - fmarty@u-bourgogne.fr; Nathalie Leborgne-Castel* - lcastel@u-bourgogne.fr \\ * Corresponding author
}

Published: 04 August 2005

BMC Plant Biology 2005, 5:13 doi:10.1186/147|-2229-5-13
Received: 08 April 2005

Accepted: 04 August 2005

This article is available from: http://www.biomedcentral.com/I47I-2229/5/I3

(C) 2005 Reisen et al; licensee BioMed Central Ltd.

This is an Open Access article distributed under the terms of the Creative Commons Attribution License (http://creativecommons.org/licenses/by/2.0), which permits unrestricted use, distribution, and reproduction in any medium, provided the original work is properly cited.

\begin{abstract}
Background: The vegetative plant vacuole occupies $>90 \%$ of the volume in mature plant cells. Vacuoles play fundamental roles in adjusting cellular homeostasis and allowing cell growth. The composition of the vacuole and the regulation of its volume depend on the coordinated activities of the transporters and channels localized in the membrane (named tonoplast) surrounding the vacuole. While the tonoplast protein complexes are well studied, the tonoplast itself is less well described. To extend our knowledge of how the vacuole folds inside the plant cell, we present three-dimensional reconstructions of vacuoles from tobacco suspension cells expressing the tonoplast aquaporin fusion gene BobTIP26-1::gfp.

Results: 3-D reconstruction of the cell vacuole made possible an accurate analysis of large spanning folds of the vacuolar membrane under both normal and stressed conditions, and suggested interactions between surrounding plastids. Dynamic, high resolution 3-D pictures of the vacuole in tobacco suspension cells monitored under different growth conditions provide additional details about vacuolar architecture. The GFP-decorated vacuole is a single continuous compartment transected by tubular-like transvacuolar strands and large membrane surfaces. Cell culture under osmotic stress led to a complex vacuolar network with an increased tonoplast surface area. In-depth 3-D realistic inspections showed that the unity of the vacuole is maintained during acclimation to osmotic stress. Vacuolar unity exhibited during stress adaptation, coupled with the intimate associations of vacuoles with other organelles, suggests a physiological role for the vacuole in metabolism, and communication between the vacuole and organelles, respectively, in plant cells. Desiccation stress ensuing from PEG treatment generates "double" membrane structures closely linked to the tonoplast within the vacuole. These membrane structures may serve as membrane reservoirs for membrane reversion when cells are reintroduced to normal growth conditions.
\end{abstract}

Conclusion: 3-D processing of a GFP-labeled tonoplast provides compelling visual constructions of the plant cell vacuole and elaborates on the nature of tonoplast folding and architecture. Furthermore, these methods allow real-time determination of membrane rearrangements during stresses. 


\section{Background}

Space-filling, turgor-driving vacuoles must have originated at a very early stage of biological evolution and have subsequently evolved to undertake various functions, well-known in algae, fungi (including yeast), and plants [1]. Many of the advanced, complex functions operate on or are closely associated with the vacuolar sap-bounding membrane, i.e. the tonoplast. A detailed structural study of dynamic events mediated by the tonoplast should extend our knowledge of its cellular functions. In the past, in vivo observations of the vacuolar membrane were restricted by the resolution of light microscopy. Like other cell components of a size below the limit of resolution of the light microscope, the tonoplast has been studied mostly by electron microscopy. However, this permits only post-mortem observations of thin sections from rapidly frozen freeze-substituted or chemically fixed cells [2], or replicas of fracture faces from fast-frozen cells $[3,4]$. Although these studies have provided invaluable insights into the organization and biogenesis of the tonoplast, analysis of thin specimens provides only limited information about its spatial architecture. High voltage electron microscopes have the ability to retrieve large amounts of information from thick sections through fixed cells, but depend on the use of a restricted number of selective (non-vital) "staining" techniques to overcome the decrease in image contrast typically seen at high accelerating voltages [4-6]. As such, the use of these different techniques makes it difficult to study and understand the dynamic changes of the vacuolar membrane in living cells.

The confocal fluorescence microscope can eliminate outof-focus blur. Three-dimensional (3-D) data from intact biological specimens can therefore be obtained by noninvasive optical sectioning [7]. A new revolution in microscopy came over a decade ago with the use of "green fluorescent protein" (GFP) from jellyfish in vivo [8]. GFP and its variants are now frequently used to generate a fluorescent organelle, e.g. mitochondria [9], chloroplasts [10] or components of the secretory system [11], allowing one to study their dynamics in living cells. Furthermore, several studies present data of GFP-labeled tonoplasts [12-21], of which only a few followed tonoplast dynamics [20,21].

Since the best resolution of living cells is obtained with laser scanning confocal microscopy images, plant-compatible GFP cDNA [22] was fused, in frame, to the 3' end of the cauliflower BobTIP26-1 tonoplast-specific aquaporin cDNA cloned in our laboratory [23]. The resultant chimeric gene was expressed in tobacco cells (var. Wisconsin 38) grown in suspension, which display a GFP-decorated tonoplast [17]. This method of in vivo labeling was used to 3-dimensionally reconstruct the tonoplast of cells at different stages of growth and under var- ious osmotic stresses. Our data show that the GFPdecorated vacuole in tobacco suspension cells is a single continuous compartment. Furthermore, osmotic stress conditions yield a greater tonoplast surface area while maintaining vacuole unity. Moreover, PEG treatment generates spherical structures that are associated with the inner side of the vacuolar membrane. We propose that these structures serve as membrane reservoirs necessary for membrane reorganization after cells are returned to normal growth conditions.

\section{Results}

\section{Three-dimensional reconstruction of the vacuole}

We have recently expressed a tonoplast aquaporin fusion gene BobTIP26-1::gfp under the CaMV35S promoter in tobacco cells [17]. The proper targeting of the resultant fusion protein to the tonoplast was confirmed by laser scanning confocal microscopy (Additional file 1). Tobacco cells expressing the chimeric protein were used for 3-D reconstructions of the cell vacuoles.

In 7 day-old turgid cells, vacuoles occupy almost the entire cell volume. In a projection view, where 40 optical sections of such cells were merged (z-step = $1 \mu \mathrm{m}$; Fig. 1a), a 3-D relief of the vacuole was barely observable, and structural details of the tonoplast were not easily discerned. Realistic 3-D pictures of the tonoplast (Fig. 1b, 1c) were obtained after isosurface extraction, a procedure in which volume images were converted into geometric surfaces [24] by using the 3-D visualization software Imaris 2.7 (Bitplane AG, Switzerland). The intravacuolar surface of the tonoplast was readily scrutinized when only "half vacuoles" were reconstructed. As expected, red autofluorescent chloroplasts were seen at the outer surface of the GFP-labeled membranes, confirming their cytoplasmic localization (Fig. 1b). Chloroplasts were also clustered around the nucleus, where the tonoplast forms a cavity (Fig. 1, arrow). Labeled transvacuolar cytoplasmic strands were seen radiating throughout the vacuole, extending from the nuclear region to the cell periphery (Fig. 1c, arrowheads). Furrows were also occasionally observed on the cytoplasmic side of the tonoplast (Fig. 2a, arrows). When the isosurface mode was used to visualize simultaneously chloroplasts and tonoplasts within large vacuolated cells, numerous chloroplasts were seen lying within these furrows (Fig. 2b).

We observed cells of different sizes and shapes in the nonsynchronized tobacco cell suspension. While confocal images suggest the existence of several vacuoles inside each cell (Fig. 3a, 3c, 3e), 3-D representations of each cells' tonoplast, and the visualization of openings within the vacuolar lumens clearly support a "one cell, one vacuole" model, i.e. vacuolar continuity exists between GFPlabeled vacuolar compartments within a cell (Fig. 3b, 3d, 

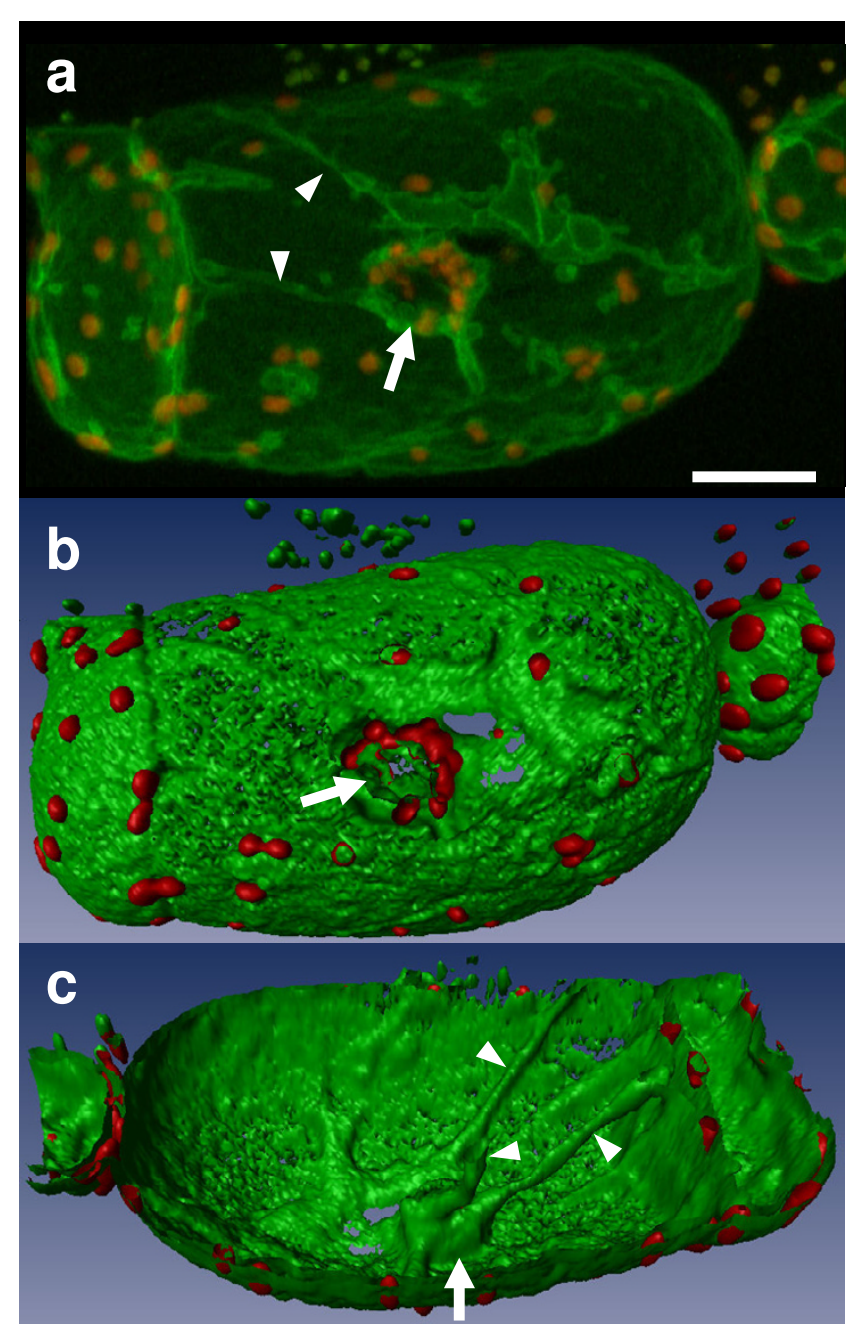

Figure I

Three-dimensional vacuole reconstruction of a vacuolated BobTIP26-I::gfp expressing cell 7 days after subculture. (a) Projection view of 40 confocal serial pictures corresponding to the half depth of the cell (i.e. $40 \mu \mathrm{m}$ ). Bar $=25 \mu \mathrm{m}$. (b) 3-D view after isosurface extraction showing the protoplasmic side of the vacuole. (c) Interior view of the vacuole after isosurface extraction. Green and red correspond to the tonoplast and the chloroplasts, respectively. Arrow: nuclear pouch; arrowheads: transvacuolar strands. The missing domains of the tonoplast surface in (b) and (c) result from an under-sampling of confocal images. The rendering of a completely smooth 3-D view would have required use of additional intermediate sections.

3f). Indeed, large surface areas of tonoplast may transect the vacuole, but gaps exist, allowing continuity of the vacuolar interior. The three joined cells (Fig. 3c) each possesses such transvacuolar layers, but 3-D representations show that they do not define discrete vacuoles in each cell

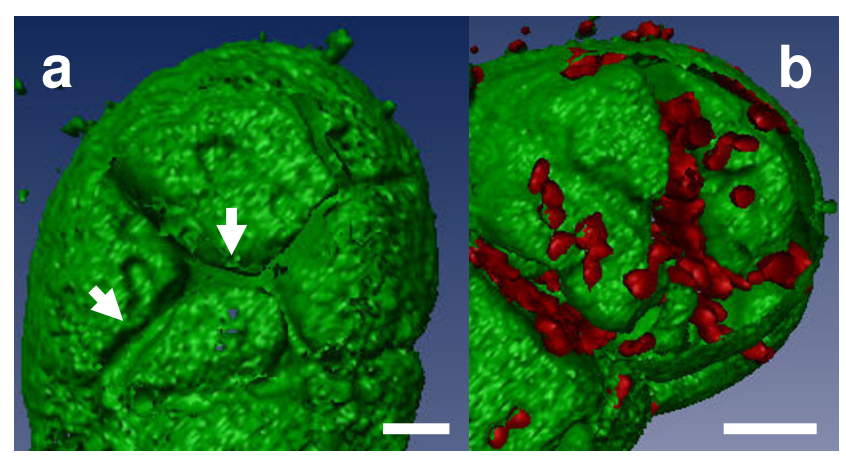

Figure 2

Outside shape of a vacuole. (a) Furrows on the outer part of the tonoplast (arrows) revealed by 3-D surface rendering. (b) Chloroplasts - in red - fill up the furrows on the tonoplast surface. Isosurfaces of both channels are displayed. $\mathrm{Bar}=10 \mu \mathrm{m}$.

(Fig. 3d). An animated sequence of these 3-D reconstructed vacuoles is shown in the Additional File 2. What seemed to be individual vacuolar cavities in the cells observed by confocal microscopy was seen as a single vacuolar compartment by 3-D reconstruction. Careful analysis through a tomogram of a protoplast prepared from BobTIP26-1::gfp expressing tobacco cells is also in accord with this feature (Additional File 3).

Cellular architecture details that are evident as a result of 3-D reconstructions include the demonstration of a vacuole with numerous transvacuolar strands, outwardly radiating from the nuclear region to the cell periphery (Fig. 3e, 3f). 3-D reconstructions also showed that chloroplasts do not merely surround the nucleus; their distribution also parallels that of the transvacuolar strands.

\section{Tonoplast behavior during plasmolysis}

We further analyzed the behavior of the labeled tonoplast during plasmolysis. Tobacco suspension cells were bathed in culture media supplemented with different osmotica (170 mM sodium chloride, $0.6 \mathrm{M}$ mannitol or $0.5 \mathrm{M}$ sorbitol) to induce plasmolysis-deplasmolysis cycles. Plasmolysis occurred 30 to 60 sec after the osmoticum entered the perfusion chamber. The phenomenon preferentially started at the cell corner, with complete plasmolysis occurring after 3 to $5 \mathrm{~min}$. Two forms of plasmolysis were observed in tobacco cells, as defined by Oparka [25]. The convex form of plasmolysis was most frequently observed, which results from an even separation of the protoplast from the walls, thereby forming a symmetrical and roughly spherical protoplast (Fig. 4a). Occasionally, the concave form of plasmolysis was observed (Fig. 4b). In this form, concave pockets are formed as the plasma 

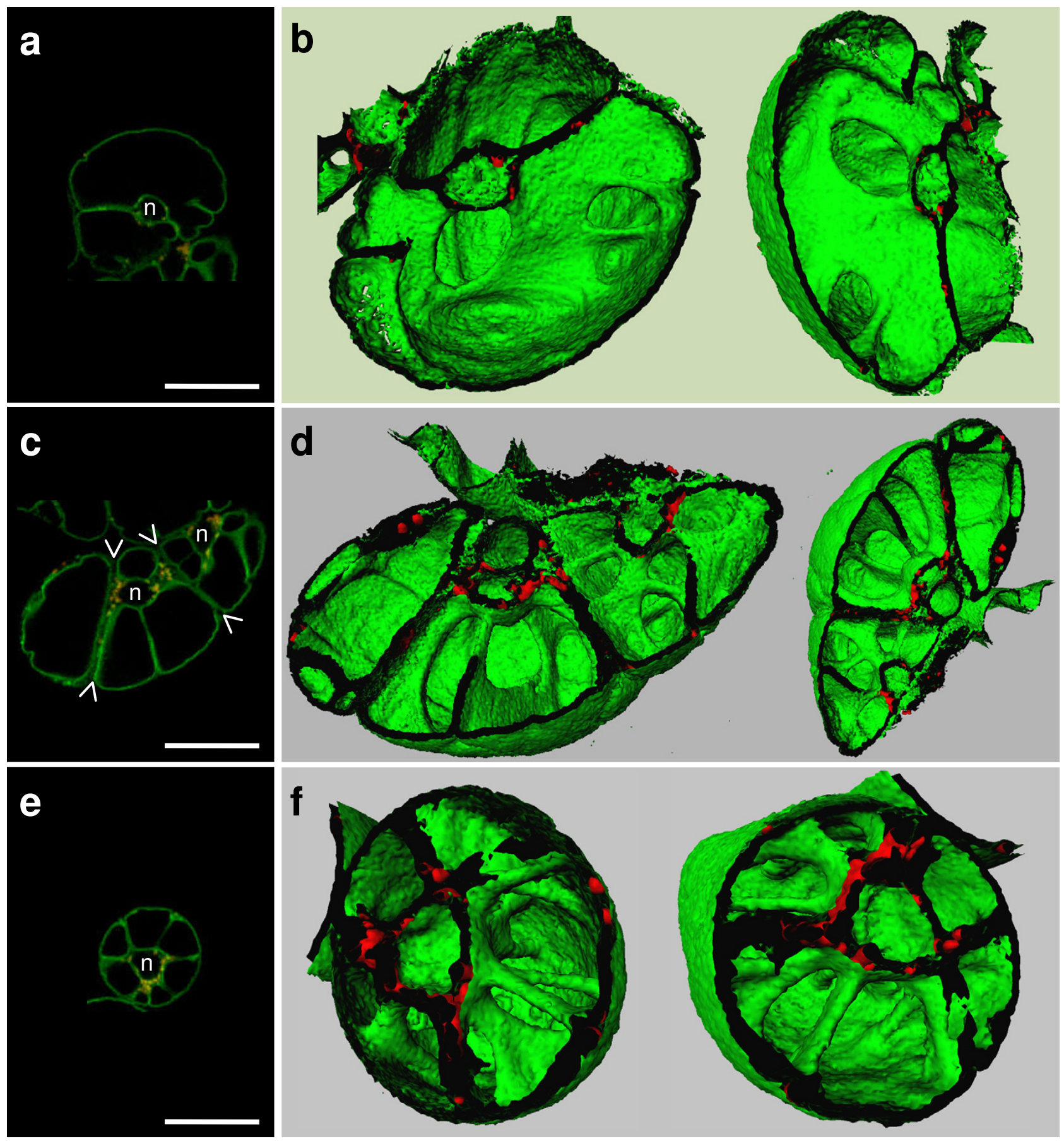

\section{Figure 3}

Vacuole continuity through gaps inside tonoplast sheets. (a, c, e) Single confocal images. The focal plane chosen corresponds to the one through the vacuoles' center. Arrowheads ( $>$ ) in (c) delimit joined cells. $n$, nuclear region. Bars $=50 \mu m$. $(b$, $d$, f) 3-D representation of the vacuole halves after isosurface extraction. Notice the openings in the vacuolar layers (b and d) and the many transvacuolar strands emanating from the nuclear region (f). 

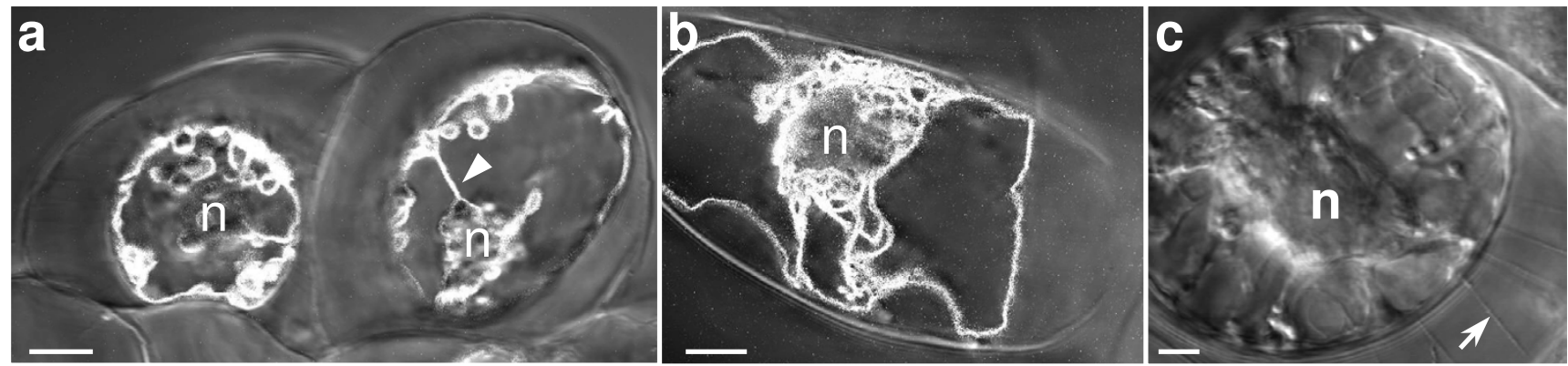

\section{Figure 4}

Cell perfusion with MS supplemented with $0.6 \mathrm{M}$ mannitol. (a) A confocal fluorescent image merged with a Nomarski interference contrast image of convex plasmolysed cells. Arrowhead: transvacuolar strand. Bar $=10 \mu \mathrm{m}$. (b) A confocal fluorescent image merged with a Nomarski interference contrast image of a concave plasmolysed cell. $\mathrm{n}, \mathrm{nucleus}$. Bar $=10 \mu \mathrm{m}$. $(\mathrm{c})$ A Nomarski interference contrast image of a plasmolysed cell. Hechtian strands (arrow) attach the protoplast tightly to the cell wall. $n$, nucleus. Bar $=5 \mu \mathrm{m}$.

membrane separates from the wall. During both form of plasmolysis, the protoplast was tightly connected to the cell wall by Hechtian strands (Fig. 4c, arrow).

During the plasmolysis step, a peculiar folding of the GFPlabeled tonoplast characterized by complex curling surrounding the nucleus developed within the vacuole (Fig. $4 a, 4 b)$. Closer examination of the curled structures revealed that they were slightly more fluorescent than the tonoplast to which they were tethered, an observation that might be due to the joining of two adjacent labeled membranes. Furthermore, the vacuole and the tonoplast remained intact and no vesicle formation was detected. A cellular tomogram through a plasmolysed cell clearly demonstrates the uninterrupted integrity of the tonoplast, its folds and curves being readily visible (Additional File 4). The absence of tonoplast inside the Hechtian strands was confirmed by the absence of labeling (Additional File 4). However, transvacuolar cytoplasmic strands could still be observed inside the vacuoles of plasmolysed (Fig. 4a, arrowhead; Additional File 5) and deplasmolysed cells (Additional file 5). These intravacuolar structures appear to be less flexible than the peripheral tonoplast. Cells were kept plasmolysed for 10 to $15 \mathrm{~min}$ before they were bathed again in normal MS culture medium. Plasmolysed cells returned to their normal shape after about 10 to 20 min (Additional File 5), and the curled structures unwound. The position of the nucleus within the cell was nearly constant throughout the plasmolysis-deplasmolysis process.

\section{Effects of osmotic stress on tonoplast architecture}

Consistent with the results obtained with 3-D reconstructions, we tried to ascertain the tonoplast architecture during osmotic stress culture. Indeed, during $\mathrm{NaCl}$ culture conditions of tobacco suspension cells, a vacuolization phenomenon, characterized by a fragmentation of the central vacuole into multiple smaller ones, has been described [26]. To prove the existence of this fragmentation in tobacco cells exhibiting a fluorescent tonoplast, BobTIP26-1::gfp expressing cells were subcultured in hyper-osmotic MS medium containing $170 \mathrm{mM} \mathrm{NaCl}, 0.6$ $\mathrm{M}$ mannitol or $0.5 \mathrm{M}$ sorbitol, with final water potentials of -1.4 MPa; -1.6 MPa and -1.2 MPa, respectively. Compared with cells subcultured in normal MS medium (Fig. $5 \mathrm{a}, 5 \mathrm{~b})$, cells acclimated in hyperosmotic medium were smaller (Fig. 5c, 5d). [Hereafter, cells which have been grown in media containing osmoticum are referred to as "acclimated cells"]. The extrapolated volumes of cells grown in normal MS and in hyperosmotic medium are on average $175 \cdot 10^{3} \mu \mathrm{m}^{3}$ and $53 \cdot 10^{3} \mu \mathrm{m}^{3}$, respectively. After acclimation, cell volume decreases $\sim 3.3$ times. If the vacuole represents $90 \%$ of the cell volume in cells grown in normal MS medium, the original vacuole volume would be $157 \cdot 10^{3} \mu \mathrm{m}^{3}$.

The vacuolization phenomenon could be monitored under Nomarski contrast interference optics (Fig. 5c). Furthermore, this phenomenon was more easily observed with fluorescent labeling of the tonoplast (Fig. 5d). In these cultures, only $25 \%$ of the cells were completely plasmolysed (Fig. 5c arrows), and did not show any fluorescence, indicating that they are non-acclimated, dead cells (Fig. 5d arrows). Additionally, vital staining with neutral red showed that only acclimated cells contained the dye in their vacuoles (data not shown).

To determine if the apparently numerous, independent, small vacuoles of acclimated cells are part of a continuous vacuolar compartment, 3-D reconstructions of the entire 

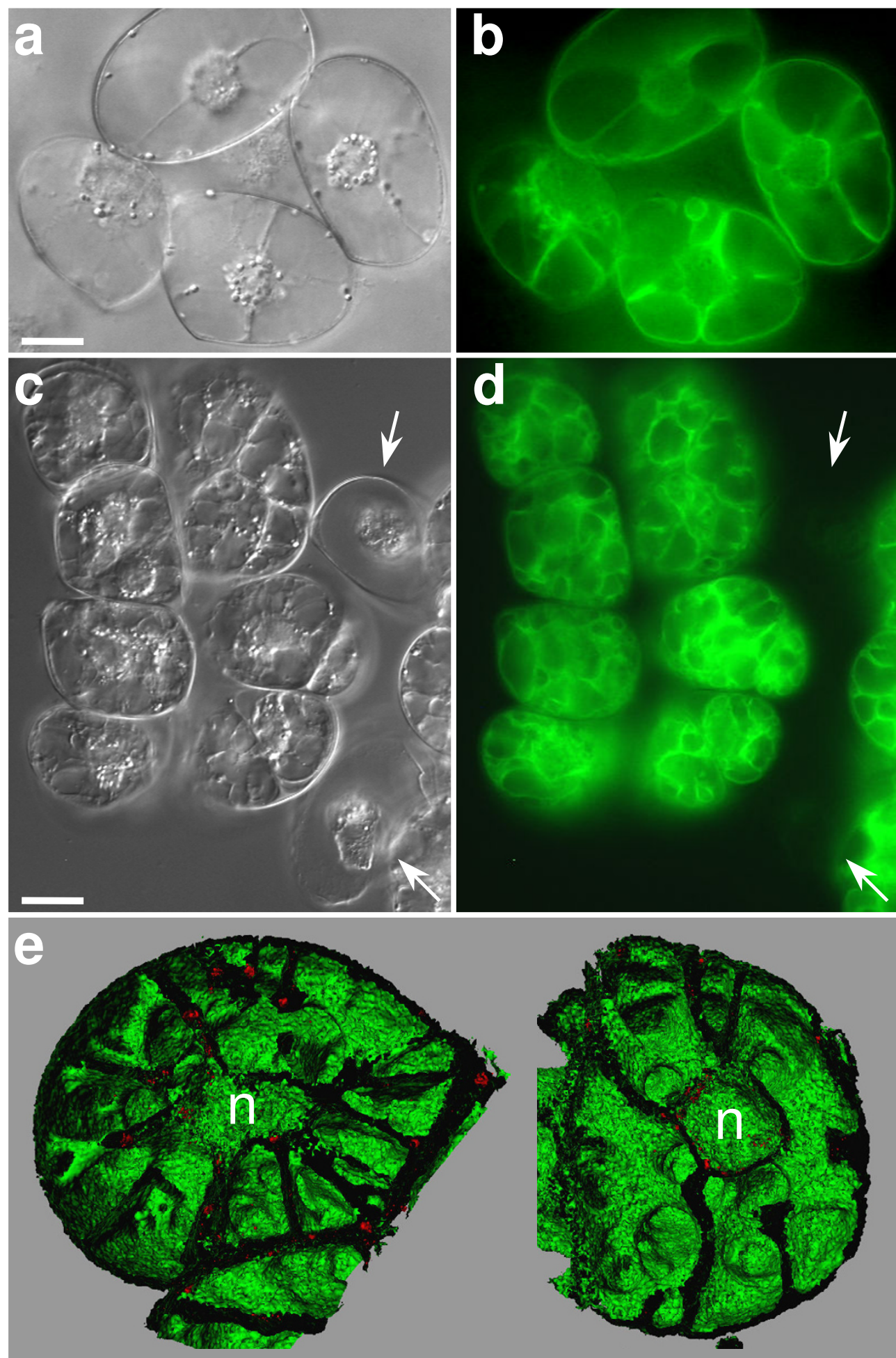

\section{Figure 5}

Normal cells versus osmotic stressed cells. (a, b) Cells under normal growth conditions. Bar $=25 \mu \mathrm{m}$. (c, d) Cells grown in MS supplemented with $0.6 \mathrm{M}$ mannitol (i.e. acclimated cells). Arrows correspond to dead cells (i.e. non-acclimated cells). Bar $=25 \mu \mathrm{m}$. (e) 3-D reconstruction of the vacuole after isosurface extraction from acclimated cells cultured in MS supplemented with $0.5 \mathrm{M}$ sorbitol. 
vacuole were achieved. The images indicated the existence of a complex vacuolar network. A depth inspection with stereo-viewers showed interconnections between the vacuolar cavities in completely reconstructed vacuoles. Halves of such reconstructed vacuoles are presented in Figure 5e. The nuclear pouch can be seen at the cell center, surrounded by small, interconnected vacuolar cavities. Despite strong evidence for the existence of a solitary vacuolar space, we cannot rule out the possibility that acclimated cells contain small, discrete cavities. However, no small structures of this nature could be discerned using the complicated reconstructions. Since vacuolar cavities are interconnected, we suggest that the tonoplast surface area increases significantly while the continuity of the vacuolar lumen remains unaltered. The software (Imaris 2.7) used for the 3-D reconstructions did not allow quantification of the tonoplast surface area, nor comparison of the tonoplast surfaces of acclimated cells to those of normal cultured cells.

\section{Tonoplast architecture modifications during dehydrative stress}

Dehydrative stress was mimicked using the macromolecule polyethylene glycol $\left(\mathrm{PEG}_{8000}\right)$, which passes with either difficulty or not at all through the cell wall [27], thereby reducing the extracellular free water concentration [28]. While an overall increase in membrane surface area occurred in hyperosmotically stressed suspension cells, cells cultured in MS medium, supplemented with $10 \%$ $\mathrm{PEG}_{8000}$, displayed numerous spherical fluorescent structures of $5-10 \mu \mathrm{m}$ in diameter, but neither complex membrane rearrangement nor folding. These inner vacuolar structures exhibited a brighter membrane fluorescence compared to that of the peripheral tonoplast (Fig. 6a, 6b, arrowheads). Indeed, the fluorescence intensity of the membrane surrounding these structures was at least twice that of the peripheral tonoplast (Fig. 6c). These spherical structures were also dynamic, moving inside the vacuolar lumen, but seemingly attached to the peripheral vacuolar membrane (Fig. 6d, arrowhead). They were easily distinguishable from the transvacuolar strands, which maintained their morphology (Fig. 6e, arrow). Indeed, the transvacuolar strands transect the lumen of the vacuole from one part of the vacuole to the other, while the spherical structures are tethered to the vacuole perimeter.

\section{Discussion}

3-D reconstruction of the vacuole under native conditions The plant vacuole is a multi-functional organelle [1] which serves as a true milieu intérieur [29], playing key roles during cell growth [30], and possibly in the osmoregulation of water during osmotic stress of the cytoplasm [31]. For the purpose of redefining the vacuole architecture, we present the use of 3-D reconstructions of the vacuolar apparatus from cells containing a GFP- labeled tonoplast under "native" conditions. The tonoplast marker system [17] was developed to gain new insights of the tobacco cell vacuole morphology during both normal and osmotic stress growth conditions. A 3-D representation of the vacuole using confocal serial pictures offers the most accurate illustration of how a vacuole is folded within a plant cell. Indeed, previous examination of mitotic BY-2 cells expressing a GFP-labeled syntaxin yielded comprehensive images of vacuolar architecture by reconstructing 3-D surfaces obtained from sequential confocal sections [32]. A side effect of the morphology of the tonoplast due to the over-expression of a membrane protein such as BobTIP26-1::GFP can, a priori, not be excluded, but results obtained for both 35S-GFPAtVam3p and the lipophilic probe FM4-64 in BY-2 cells revealed that vacuolar morphology was not artificially affected in transgenic BY-2 cells [32]. The fluorescent tonoplast in BobTIP26-1::gfp expressing cells appears to be similar to the FM4-64 stained one in BY-2 cells, assuming that vacuolar morphology in BobTIP26-1::gfp expressing cells is not altered.

The software used for the representation of the laser confocal microscope data offered two forms of volume visualization: isosurface rendering and direct volume rendering [33]. We chose the isosurface rendering method, in that it allowed us to represent a shaded surface as an easily interpretable 3-D object. However, the two techniques are complementary and the conclusions offered by the final 3-D pictures are equivalent.

A 3-D vacuole structure of BY-2 tobacco cells expressing TIP::gfp was first established by Mitsuhashi and co-workers [13], but their representation was mainly a projection view of sequential confocal images that revealed the presence of several large vacuoles folded within the cell. However, as demonstrated in the present study, a projection view may not be as informative as 3-D reconstruction (Fig. 1, Fig. 3). Whereas projection views showed multiple vacuoles folded within a single cell, 3-D reconstructions accurately portray a single vacuolar continuum within cells cultivated under normal growth conditions (Fig. 3). Such continuity of the vacuolar lumen has been described by Palevitz and co-workers [34], who analyzed the vacuole during cell differentiation of Allium stomata cells. Although thin sections of these fixed cells observed by electron microscopy revealed individual small vacuoles, a 3-D reconstruction using 0.25 to $0.50 \mu \mathrm{m}$ thick serial sections, viewed at $100 \mathrm{kV}$, clearly showed the sections originated from a continuous reticulate network.

The concept of a continuous vacuole is important for the plant cell because the content of the vacuolar sap can thereby flow between all regions of the cell when homeostatic measures are undertaken by stressed cells. 


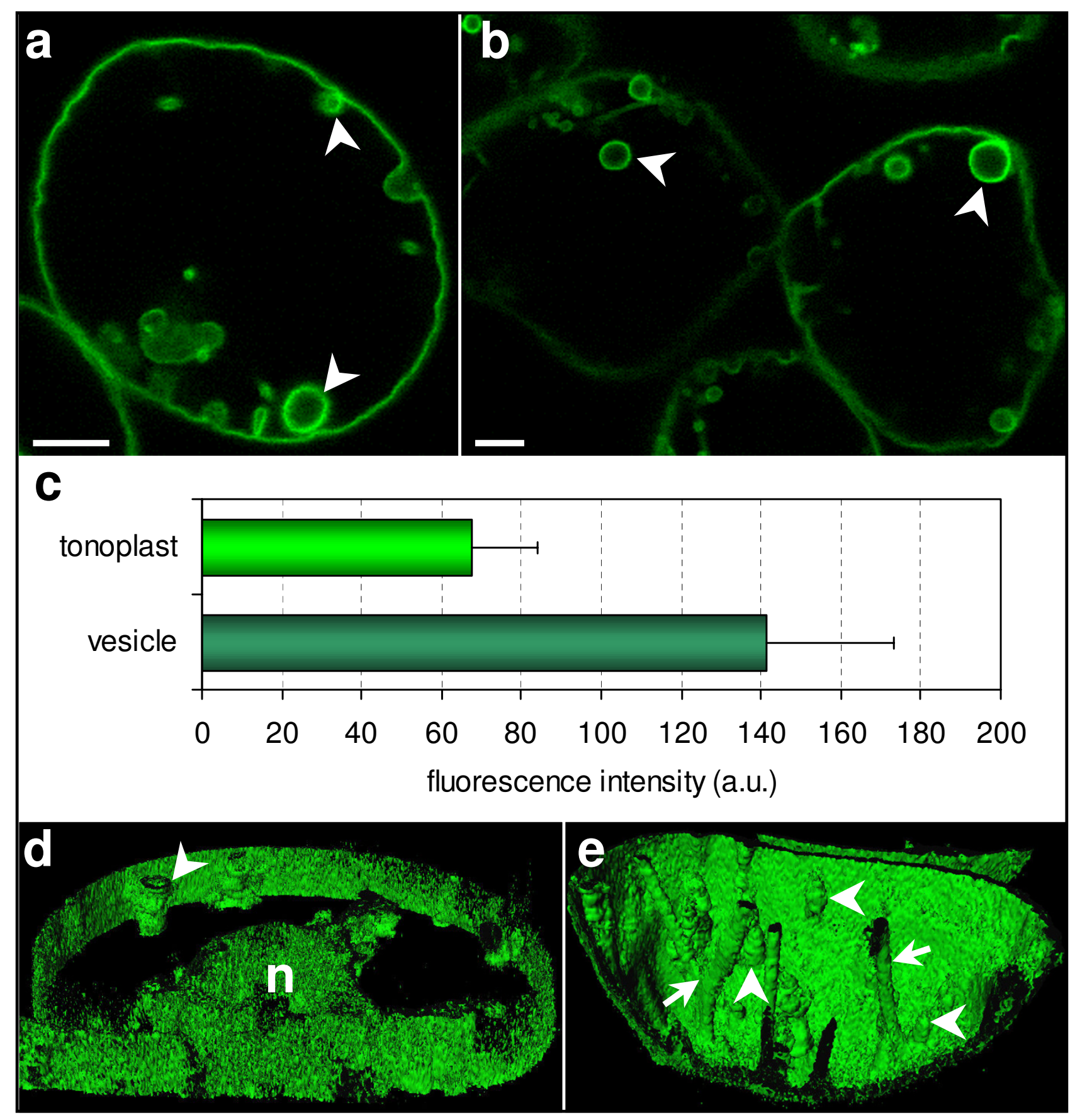

Figure 6

PEG-stressed cells. $(\mathrm{a}, \mathrm{b})$ Localization of BobTIP26- I::GFP in the membrane of spherical structures (arrowheads) within vacuoles. Bar $=10 \mu \mathrm{m}$. (c) A histogram of fluorescent intensity values collected from both the tonoplast of the cell periphery and vesicles. (d) A 3-D slice reconstruction through the vacuole at the position of the nucleus (n). Arrowhead = spherical structure. (e) 3-D reconstruction through a part of a vacuole. Transvacuolar strands are still present in these cells (arrow); spherical structures are primarily fixed onto the tonoplast (arrowheads). 
To obtain enhanced resolution of the vacuole, reconstructions based on electron tomograms should be used as exemplified in the data obtained for both Arabidopsis thaliana mitotic cell plate formation [35-37] and the Golgi apparatus of animal [38] and yeast [39] cells.

\section{Tonoplast surface architecture}

With 3-D reconstructions of the vacuole, we were able to analyze its surface architecture from a unique perspective. Earlier 3-D renderings of the vacuole-cytoplasm interface of tobacco cell vacuoles showed ripples on the acridine orange labeled vacuole surface [40], which differ from surface furrows we have described. Indeed the vacuole ripples dip into the cytoplasm, but not into the vacuole [40]. We observed the furrows just after isosurface reconstruction and they seem to result from the pressure exerted by some organelles onto the turgid vacuole. The organelles were thus squeezed between the plasma membrane and the tonoplast creating hence the furrows on the cytoplasmic interface with the vacuole. We show here that the 3-D rendering is also useful to visualize interactions between organelles.

\section{The tonoplast surface is enlarged during osmotic stress} Although membranes are targets of stress-induced cellular damage, and vacuoles are thought to have a central role during stress, few studies of the tonoplast during stress conditions have been reported. We reasoned that scrutinizing the pattern of the tonoplast during different osmotic stresses would build upon our current knowledge of how the cell organizes this membrane during environmental changes. Glycophytic cells, such as tobacco cells (Nicotiana tabacum var. Wisconsin 38), were previously analyzed for their ability to acclimate to $\mathrm{NaCl}$ [41] and determined to have an increased vacuolization as well as an extensive network of transvacuolar membrane strands [26]. Transvacuolar strands are easily visualized with Normarski optics under a light microscope; however, their composition is more difficult to deduce. Our use of the tonoplast intrinsic GFP-tagged protein that we developed [17] offers in situ evidence that transvacuolar strands transecting the vacuole lumen also contain tonoplast associated molecules (Fig. 1, Fig. 3f).

Tobacco cells growing in the presence of diverse osmotica developed an extensive membrane network that appears to transect the vacuole, thereby creating multiple compartments. Observations of similar phenomena were previously described for cells grown during $\mathrm{NaCl}$ acclimation [26]. With a 3-D representation, we found that growth in media supplemented with salt, mannitol or sorbitol resulted in transvacuolar strands completely changing their organizational pattern, shifting from a thin shape during normal conditions to larger surface areas. Concomitant with strand pattern alterations was the appearance of several small cavities. These phenomena were observed regardless of the osmoticum used for acclimation. The 3-D picture obtained from acclimated cells clearly demonstrates that the number of vacuoles remains constant while the total tonoplast surface area increases, thereby creating a more complex vacuolar pattern (Fig. $5 e$ ). Thus, the ratio of tonoplast surface area to volume of cytoplasm is optimized for exchanges (transport of water and ions) between the cytosol and the vacuole. As the total surface area of tonoplast membrane increases, it follows that the total mass of membrane compounds also increases. It is notable that similar vacuolization changes were observed to occur after hyper-osmotic treatment of wild type tobacco cells; therefore, the increased membrane surface is linked neither to the presence of the aquaporin nor the greater cell size of BobTIP26-1::gfp expressing cells [17]. Measurements of the surface areas of the tonoplasts in acclimated cells are complicated by the absence of vacuole shape uniformity. In contrast, the prolate spheroid shape of Vicia faba guard cells allows for the determination of both their surface areas and volumes after 3-D reconstruction efforts [42]. In our studies such standardization of conditions was not possible.

\section{Folding of the plasmolytic vacuole}

Notwithstanding the massive changes in vacuolar surface area which accompany plasmolysis, only a few observations on the fate of the vacuole during osmotic contraction have been described in the relevant literature. Plasmolysis-deplasmolysis cycles were realized in a perfusion chamber, allowing observation of fluorescent tonoplast labeled with GFP. During plasmolysis, it was observed that the tonoplast undergoes folding and that the vacuole does not vesiculate into discrete multilamellar vesicles severed from the tonoplast as "sac-like, rod-like or doughnut-shaped structures" as previously described [43]. Furthermore, complete tomography of a plasmolysed cell (Additional File 4) demonstrated that the tonoplast folds in a peculiar way inside the protoplast, rather than being broken into small vesicles, as reported earlier [25]. Such a process seems better suited than vesicle formation for a faster reestablishment of the vacuole during deplasmolysis. No membrane fusion is necessary.

Transvacuolar strands, thin tubular structures that traverse the vacuole, were observed not to break down both during plasmolysis and after deplasmolysis (Additional File 5). Earlier studies showed that transvacuolar strands were stabilized by actin filaments $[44,45]$ and rearranged by myosin motors through their interactions with actin filaments [46]. Despite these observation of strand stability, they have also been described as dynamic and delicate [26]. Our data support the notion that transvacuolar strands exhibit both "strength" and stability, in that their 
positions remaining static inside the vacuole during abrupt environmental changes (Additional File 5).

\section{PEG treatment results in spherical structures composed of tonoplast}

Surprisingly, spherical structures with twice the fluorescent intensity as the tonoplast were observed inside the vacuole lumen of tobacco suspension cells cultured in MS medium supplemented with $10 \% \mathrm{PEG}_{8000}$. These structures were similar to those frequently observed in either tonoplast GFP-labeled tobacco leaves $[47,48]$ or in Arabidopsis cotyledons [49]. Additionally, such structures have been described in cotyledons, hypocotyls and roots of the Arabidopsis vacuolar biogenesis bub (bubble-bath) mutants [50], as well as in transiently transformed Nicotiana benthamiana plants expressing a GFP fusion protein homologous to a high-affinity tonoplast phosphate transporter [51]. The structures we observed during PEG treatment were independent of the water regulation or overexpression of an aquaporin gene because we, as well as other authors, found similar bulbs in Arabidopsis transformed cells with native TIP1;1 promoter (Bouhidel K., personal communication) and in cells expressing other tonoplast proteins under the control of the 35 S promoter [51]. The structures observed in Arabidopsis cotyledons [49] were called "bulbs", a term which reflects a spherical structure that is not entirely closed. The open section of these bulbs may be the result of incomplete vesicle formation [49]. The bulbs appear to be attached to the actin cytoskeleton, as actin inhibitor treatment allowed their immobilization and 3-D reconstructions [51]. The 3-D images of the spherical structures we observed in tobacco suspension cells cultured under dehydration stress are similar to the 3-D reconstructed cylindrical structures observed in GFP-AtVam3p expressing root protoplasts of A. thaliana [52]. Recently, fluorescent circular structures were seen in germinating pollen tubes of $\delta$-TIP::GFP expressing Arabidopsis plants [20]. These mobile cytoplasmic invaginations may be a widespread characteristic of actively growing tissues.

Why do vacuoles of PEG acclimated suspension cells contain "bulbs"? PEG mimics dehydration when applied to the culture medium, resulting in a decrease of turgor. Similarly, cotyledons undergo a desiccation process. In Spirodela intermedia upper mesophyll cells, a breakdown of the tonoplast into small vesicles was observed after PEG inclusion [53]. Correlation of observed membrane fluorescence intensity with protein quantity suggests that the membrane of the spherical vacuolar structures is likely to contain twice the amount of aquaporins per surface area as the peripheral tonoplast, suggesting a higher level of water exchange. The spherical structures could reflect the presence of lipid domains in the tonoplast where aquaporins are concentrated and vesicle formation occurs. Indeed, some studies showed a high tonoplast fluidity [54], as well as a specific feature of fatty acid composition that may be responsible for the tonoplasts' unique fluidity and high elasticity [55] required for osmotic processes in the cell. Our results support the invagination model, postulated by Uemura and co-workers [52], where parts of the tonoplast form a double-layered membrane structure inside the vacuolar lumen. The spherical structures could serve as a reservoir, not only for membrane expansion, but also to allow for quicker homeostasis adjustments. A higher tonoplast surface area to cell volume ratio would greatly enhance the cells' capacity to maintain large ion pools during growth [56].

\section{Conclusion}

The data presented in this study demonstrates the utility of the aquaporin BobTIP26-1::GFP as a powerful tool for visualizing 3-D membrane rearrangements within stressed tobacco cells. The technique used here provides highly resolved pictures and support of the notion that tobacco suspension cells contain a single major vacuole with a lytic function. Further investigations are required to establish the exact origin and function of the membrane enclosed, intra-vacuolar circular structures exhibited by tobacco cells under PEG mediated stress.

\section{Methods \\ Plant material}

Tobacco (Nicotiana tabacum L. var. Wisconsin 38) suspension cells expressing BobTIP26-1::gfp [17] were grown in MS medium [57], with regular shaking, at $24^{\circ} \mathrm{C}$ under constant photosynthetic illumination $\left(200 \mu \mathrm{E} \cdot \mathrm{m}^{-2} \cdot \mathrm{sec}^{-}\right.$ $\left.{ }^{1}\right)$.

\section{Three-dimensional reconstruction}

Three to seven day-old cells were analyzed under a Leica TCS 4D laser confocal microscope (Leica Microsystems, Wetzlar, Germany) equipped with an argon-krypton laser (488/515 BP-FITC). The laser was focused on individual cells through a 40x NA1 oil-immersion objective. For each cell, a stack of between 100 and 200 images was collected (resolution $256 \times 256$ with 0.50 to $0.65 \mu \mathrm{m}$ of z-step). Merged individual confocal images (red and green channels) were composed using Corel Photo-Paint 7 software (Corel, Ottawa, Canada). To obtain 3-D reconstructions, confocal image stacks were imported into the threedimensional visualization software Imaris 2.7 (Bitplane AG, Switzerland) running on a Silicon Graphics ${ }^{\circledast}$ Octane $2^{\mathrm{TM}}$ workstation (SGI, Paris, France). After baseline subtraction, a subregion was defined. Then, the isosurface module of Imaris was used to reconstitute the 3-D pictures. An adequate isovalue was defined for each channel prior to viewing of the computed surface using IvView. Isosurface rendered pictures were then stored as tiff files using the MediaRecorder media tool. Realistic on-screen 
representations of 3-D reconstructions were analyzed in depth with stereoscopic viewing devices (CrystalEyes ${ }^{\circledR}$, StereoGraphics ${ }^{\circledast}$ ).

\section{Cell perfusion}

Cell perfusion was performed in a homemade perfusion chamber linked to a peristaltic pump (flux $=20 \mathrm{~mL} \cdot \mathrm{h}^{-1}$ ) (IBMP, Strasbourg, France). BobTIP26-1::gfp expressing cells were first adhered to poly-lysinized glass cover slips. The following solutions were used for cell perfusions: MS supplemented with either $0.17 \mathrm{M} \mathrm{NaCl}$ or $0.6 \mathrm{M}$ mannitol or $0.5 \mathrm{M}$ sorbitol. Plasmolysis and deplasmolysis were monitored using an inverted laser confocal microscope LSM510 (Zeiss Axiovert 100 M, Jena, Germany) fitted with a Zeiss $63 \mathrm{X}$ water-immersion objective. Confocal time lapse series were then collected. Osmolarities were measured with a Vapro ${ }^{\circledR}$ vapor pressure osmometer (Model 5520; Wescor, Logan, UT, USA).

\section{Acclimation stress conditions}

Five mL of BobTIP26-1::gfp expressing cells at exponential growth phase, were subcultured in MS medium supplemented with any of the following osmotica: $170 \mathrm{mM}$ $\mathrm{NaCl}, 0.6 \mathrm{M}$ mannitol, $0.5 \mathrm{M}$ sorbitol or $10 \% \mathrm{PEG}_{8000}$. The stressed and control (MS without an osmoticum) cells were analyzed over 7 days and 3-D reconstructed as described above. Fluorescent intensities were measured using IPLab software (Scanalytics, Fairfax, VA, USA).

\section{Authors' contributions}

DR drafted the manuscript, carried out the experiments, including the microscopic observations and computer generated reconstructions. FM and NLC conceived of the study, and participated in its design and coordination. NLC also helped to draft the manuscript. All authors read and approved the final manuscript.

\section{Additional material}

\section{Additional File 1}

Cellular tomogram through two overlapping BobTIP26-1::gfp expressing cells.

Click here for file

[http://www.biomedcentral.com/content/supplementary/14712229-5-13-S1.mov]

\section{Additional File 2}

Animation of 3-D reconstructed vacuoles. The reconstructed vacuoles from Figure $3 d$ were rotated. Notice chloroplasts (red) on the outer sides of the vacuoles, within transvacuolar strands and the cells' peripheries. Click here for file

[http://www.biomedcentral.com/content/supplementary/14712229-5-13-S2.mov]

\section{Additional File 3}

Cellular tomogram through a protoplast with a GFP-labeled tonoplast. The protoplast was prepared from BobTIP26-1::gfp expressing cells as previously described [17].

Click here for file

[http://www.biomedcentral.com/content/supplementary/1471-

2229-5-13-S3.mov]

\section{Additional File 4}

Cellular tomogram through a plasmolysed cell demonstrating the intact tonoplast structure and its folding. The tonoplast fluoresces in green. Hechtian strands are not labeled. Bar $=5 \mu \mathrm{m}$.

Click here for file

[http://www.biomedcentral.com/content/supplementary/14712229-5-13-S4.mov]

\section{Additional File 5}

Plasmolysis-deplasmolysis cycle of cells bathed with $0.5 \mathrm{M}$ sorbitol. Time is marked at the bottom left; changes of media are given at the top left (10 min: + 0.5 M sorbitol; 23.5 min: + normal MS medium $)$. Bar $=$ $20 \mu \mathrm{m}$.

Click here for file

[http://www.biomedcentral.com/content/supplementary/14712229-5-13-S5.mov]

\section{Acknowledgements}

The authors would like to thank J.C. Robbins for English corrections and meaningful discussions. D.R. was supported by the Ministère de l'Education Nationale de la Recherche et de la Technologie ( $N^{\circ}$ 99-5-12166). The Octane $2^{\mathrm{TM}}$ workstation and the Imaris software were used at the CMABSERCOBIO (Université de Bourgogne). We thank C. Ritzenthaler at The Inter-Institute Confocal Microscopy Plate-Form in the Institut de Biologie Moléculaire des Plantes (Strasbourg) for his assistance with the perfusion experiments. The Conseil Régional de Bourgogne financed this work.

\section{References}

I. Marty F: Plant Vacuoles. Plant Cell 1999, I I:587-600.

2. Buvat R: Electron microscopy of plant protoplasm. Int Rev Cytol 1963, 14:41-55.

3. Fineran BA: Association between endoplasmic reticulum and vacuoles in frozen-etched root tips. J Ultrastruct Res 1973, 43:75-87.

4. Marty F: High voltage electron microscopy of membrane interactions in wheat. J Histochem Cytochem 1980, 28: I I29- I I 32.

5. Cosslet VE: The "ultrastructure" of tissues. Revelations by electron-microscope. Radiography 1962, 28:203-204.

6. Favard $\mathrm{P}$, Cassano $\mathrm{N}$ : The preparation and observation of thick biological sections in the high voltage electron microscope. J Microsc 1973, 97:59-8I.

7. Amos WB, White JG: How the confocal laser scanning microscope entered biological research. Biol Cell 2003, 95:335-342.

8. Tsien RY: The green fluorescent protein. Annu Rev Biochem 1998, 67:509-544.

9. Köhler RH, Zipfel WR, Webb WW, Hanson MR: The green fluorescent protein as a marker to visualize plant mitochondria in vivo. Plant J 1997, I I:613-621.

10. Köhler RH, Cao J, Zipfel WR, Webb WW, Hanson MR: Exchange of protein molecules through connections between higher plant plastids. Science 1997, 276:2039-2042.

II. Brandizzi F, Irons SL, Johansen J, Kotzer A, Neumann U: GFP is the way to glow: bioimaging of the plant endomembrane system. J Microsc 2004, 2 1 4:138-158.

12. Ueoka-Nakanishi H, Tsuchiya T, Sasaki M, Nakanishi Y, Cunningham $\mathrm{KW}$, Maeshima M: Functional expression of mung bean $\mathrm{Ca2+l}$ 
$\mathrm{H+}$ antiporter in yeast and its intracellular localization in the hypocotyl and tobacco cells. Eur J Biochem 2000, 267:3090-3098.

13. Mitsuhashi N, Shimada T, Mano S, Nishimura M, Hara-Nishimura I: Characterization of organelles in the vacuolar-sorting pathway by visualization with GFP in tobacco BY-2 cells. Plant Cell Physiol 2000, 41:993-1001.

14. Czempinski K, Frachisse JM, Maurel C, Barbier-Brygoo H, MuellerRoeber B: Vacuolar membrane localization of the Arabidopsis 'two-pore' K+ channel KCOI. Plant J 2002, 29:809-820.

15. Kutsuna N, Hasezawa S: Dynamic organization of vacuolar and microtubule structures during cell cycle progression in synchronized tobacco BY-2 cells. Plant Cell Physiol 2002, 43:965-973.

16. Yamaguchi H, Nishizawa NK, Nakanishi H, Mori S: IDI7, a new ironregulated $A B C$ transporter from barley roots, localizes to the tonoplast. J Exp Bot 2002, 53:727-735.

17. Reisen D, Leborgne-Castel N, Özalp C, Chaumont F, Marty F Expression of a cauliflower tonoplast aquaporin tagged with GFP in tobacco suspension cells correlates with an increase in cell size. Plant Mol Biol 2003, 52:387-400.

18. Yano K, Matsui S, Tsuchiya T, Maeshima M, Kutsuna N, Hasezawa S, Moriyasu Y: Contribution of the plasma membrane and central vacuole in the formation of autolysosomes in cultured tobacco cells. Plant Cell Physiol 2004, 45:95I-957.

19. Kataoka T, Watanabe-Takahashi A, Hayashi N, Ohnishi M, Mimura T, Buchner P, Hawkesford MJ, Yamaya T, Takahashi H: Vacuolar sulfate transporters are essential determinants controlling internal distribution of sulfate in Arabidopsis. Plant Cell 2004, 16:2693-2704.

20. Hicks GR, Rojo E, Hong S, Carter DG, Raikhel NV: Geminating pollen has tubular vacuoles, displays highly dynamic vacuole biogenesis, and requires VACUOLESSI for proper function. Plant Physiol 2004, 134: I227-I 239

21. Saito C, Morita MT, Kato T, Tasaka M: Amyloplasts and vacuolar membrane dynamics in the living graviperceptive cell of the Arabidopsis inflorescence stem. Plant Cell 2005, 17:548-558.

22. Haseloff J, Siemering KR, Prasher DC, Hodge S: Removal of a cryptic intron and subcellular localization of green fluorescent protein are required to mark transgenic Arabidopsis plants brightly. Proc Natl Acad Sci USA 1997, 94:2 I 22-2 27.

23. Barrieu F, Thomas D, Marty-Mazars D, Charbonnier M, Marty F: Tonoplast intrinsic proteins from cauliflower (Brassica oleracea $L$. var. botrytis): immunological analysis, cDNA cloning and evidence for expression in meristematic tissues. Planto 1998, 204:335-344.

24. Morgan F, Barbarese E, Carson JH: Visualizing cells in three dimensions using confocal microscopy, image reconstruction and isosurface rendering: application to glial cells in mouse central nervous system. Scanning Microsc 1992 6:345-357.

25. Oparka KJ: Plasmolysis: new insights into an old process. New Phytol 1994, | 26:57|-59|.

26. Chang PFL, Damsz B, Kononowicz, Reuveni M, Chen Z, Xu Y, Hedges K, Tseng CC, Singh NK, Binzel ML, Narasimhan ML, Hasegawa PM, Bressan RA: Alterations in cell membrane structure and expression of a membrane-associated protein after adaptation to osmotic stress. Physiol Plant 1996, 98:505-5I6.

27. Baron-Epel O, Hernandez D, Jiang LW, Meiners S, Schindler M: Dynamic continuity of cytoplasmic and membrane compartments between plant cells. / Cell Biol 1988, 106:715-72I.

28. Rains DW: Plant tissue and protoplast culture: applications to stress physiology and biochemistry. In Plants under stress Edited by: Jones HG, Flowers TJ and Jones MB. Cambridge, Cambridge University Press; 1989:181-169.

29. Marty F: The biogenesis of vacuoles: insights from microscopy. In The plant vacuole Advances in Botanical Research Volume 25. Edited by: Leigh A and Sanders D. San Diego, Academic Press; 1997:1-42

30. Raven JA: The role of vacuoles. New Phytol 1987, 106:357-422.

31. Hasegawa PM, Bressan RA, Zhu JK, Bohnert HJ: Plant cellular and molecular responses to high salinity. Annu Rev Plant Physiol Plant Mol Biol 2000, 5 I : 463-499.

32. Kutsuna N, Kumagai F, Sato MH, Hasezawa S: Three-dimensional reconstruction of tubular structure of vacuolar membrane throughout mitosis in living tobacco cells. Plant Cell Physiol 2003, 44:1045-1054.
33. Razdan A, Patel K, Farin GE, Capco DG: Volume visualization of multicolor laser confocal microscope data. Comp Graph 200I, 25:37I-382

34. Palevitz BA, O'Kane DJ, Kobres RE, Raikhel NV: The vacuole system in stomatal cells of Allium vacuole movements and changes in morphology in differentiating cells as revealed by epifluorescence, video and electron microscopy. Protoplasma 1981, 109:23-55.

35. Otegui MS, Mastronarde DN, Kang BH, Bednarek SY, Staehelin LA Three-dimensional analysis of syncytial-type cell plates during endosperm cellularization visualized by high resolution electron tomography. Plant Cell 200I, I3:2033-205I.

36. Otegui MS, Staehelin LA: Electron tomographic analysis of postmeiotic cytokinesis during pollen development in Arabidopsis thaliana. Planta 2004, 21 8:50I-5I5.

37. Segui-Simarro JM, Austin JR, White EA, Staehelin LA: Electron tomographic analysis of somatic cell plate formation in meristematic cells of Arabidopsis preserved by high-pressure freezing. Plant Cell 2004, 16:836-856

38. Marsh BJ, Mastronarde DN, Buttle KF, Howell KE, Mclntosh JR Organellar relationships in the Golgi region of the pancreatic beta cell line, HIT-TI5, visualized by high resolution electron tomography. PNAS 200I, 98:2399-2406.

39. Mogelsvang S, Gomez-Ospina N, Soderholm J, Glick BS, Staehelin LA: Tomographic evidence for continuous turnover of Golgi cisternae in Pichia pastoris. Mol Biol Cell 2003, I4:2277-229I.

40. Verbelen JP, Tao W: Mobile arrays of vacuole ripples are common in plant cells. Plant Cell Rep 1998, 17:917-920.

4I. Binzel ML, Hasegawa PM, Handa AK, Bressan RA: Adaptation of tobacco cells to NaCl. Plant Physiol 1985, 79: I I8-125.

42. Shope JC, DeWald DB, Mott KA: Changes in surface area of intact guard cells are correlated with membrane internalization. Plant Physiol 2003, I33:| 3| 4-1321.

43. Johnson-Flanagan AM, Singh J: Membrane deletion during plasmolysis in hardened and non-hardened plant cells. Plant Cell Environ 1986, 9:299-306.

44. Staiger CJ, Yuan M, Valenta R, Shaw PJ, Warn RM, Lloyed CW: Microinjected profilin affects cytoplasmic streaming in plant cells by rapidly depolymerizing actin microfilaments. Curr Biol 1994, 4:215-219.

45. Kost B, Spielhofer P, Chua NH: A GFP-mouse talin fusion protein labels plant actin filaments in vivo and visualizes the actin cytoskeleton in growing pollen tubes. Plant J 1998, 16:393-401

46. Hoffmann A, Nebenführ A: Dynamic rearrangements of transvacuolar strands in BY-2 cells imply a role of myosin in remodeling the plant actin cytoskeleton. Protoplasma 2004, 224:20I-2I0.

47. Reisen D: Etude de l'expression d'un gène codant une aquaporine tonoplastique de chou-fleur chez le tabac. Analyse de la dynamique vacuolaire et de l'effet de stress osmotiques. In PhD Thesis University of Burgundy, Dijon, France; 2003.

48. Kotzer AM, Brandizzi F, Neumann U, Paris N, Moore I, Hawes C: AtRabF2b (Ara7) acts on the vacuolar trafficking pathway in tobacco leaf epidermal cells. / Cell Sci 2004, I I 7:6377-6389.

49. Saito C, Ueda T, Abe H, Wada Y, Kuroiwa T, Hisada A, Furuya M, Nakano A: A complex and mobile structure forms a distinct subregion within the continuous vacuolar membrane in young cotyledons of Arabidopsis. Plant J 2002, 29:245-255.

50. Avila EL, Zouhar J, Agee AE, Carter DG, Chary SN, Raikhel NV: Tools to study plant organelle biogenesis. Point mutation lines with disrupted vacuoles and high-speed confocal screening of green fluorescent protein-tagged organelles. Plant Physiol 2003, I33:1673-1676.

51. Escobar NM, Haupt S, Thow G, Boevink P, Chapman S, Oparka K: High-throughput viral expression of cDNA-green fluorescent protein fusions reveals novel subcellular addresses and identifies unique proteins that interact with plasmodesmata. Plant Cell 2003, 15:1507-1523.

52. Uemura T, Yoshimura SH, Takeyasu K, Sato MH: Vacuolar membrane dynamics revealed by GFP-AtVam3 fusion protein. Genes Cells 2002, 7:743-753.

53. Klich MG, Didone NG, Fernandez OA, Mujica MB: Ultrastructural changes in Spirodela intermedia in response to osmoticallyinduced water shortage. Biocell 2000, 24:85-88. 
54. Leborgne N, Dupou-Cezanne L, Teulières $\mathrm{C}$, Canut $\mathrm{H}$, Tocanne JF, Boudet AM: Lateral and rotational mobilities of lipids in specific cellular membranes of Eucalyptus gunnii cultivars exhibiting different freezing tolerance. Plant Physiol 1992, 100:705-715.

55. Makarenko SP, Konenkina TA, Salyaev RK: Characteristics of fatty acid composition of lipids in higher plant vacuolar membranes. Membr Cell Biol 2000, 13:687-695.

56. Reuveni M, Bennett AB, Bressan RA, Hasegawa PM: Enhanced H+ transport capacity and ATP hydrolysis activity of the tonoplast H+-ATPase after $\mathbf{N a C l}$ adaptation. Plant Physiol 1990, 94:524-530.

57. Murashige T, Skoog F: A revised medium for rapid growth and bioassays with tobacco tissue cultures. Physiol Plant 1962, 15:473-497.

Publish with Bio Med Central and every scientist can read your work free of charge

"BioMed Central will be the most significant development for disseminating the results of biomedical research in our lifetime. "

Sir Paul Nurse, Cancer Research UK

Your research papers will be:

- available free of charge to the entire biomedical community

- peer reviewed and published immediately upon acceptance

- cited in PubMed and archived on PubMed Central

- yours - you keep the copyright

Submit your manuscript here:

http://www.biomedcentral.com/info/publishing_adv.asp
BioMedcentral 